\title{
Broadband Reflectionless Metasheets: Frequency-Selective Transmission and Perfect Absorption
}

\author{
V. S. Asadchy, ${ }^{1,2,}{ }^{*}$ I. A. Faniayeu, ${ }^{2,3}$ Y. Ra'di, ${ }^{1}$ S. A. Khakhomov, ${ }^{2}$ I. V. Semchenko, ${ }^{2}$ and S. A. Tretyakov ${ }^{1}$ \\ ${ }^{1}$ Department of Radio Science and Engineering, Aalto University, \\ P. O. Box 13000, FI-00076 Aalto, Finland \\ ${ }^{2}$ Department of General Physics, Francisk Skorina Gomel State University, 246019 Gomel, Belarus \\ ${ }^{3}$ Research Institute of Electronics, Shizuoka University 3-5-1 Johoku, \\ Naka-ku, Hamamatsu 432-8011, Japan
}

(Received 23 February 2015; published 14 July 2015)

\begin{abstract}
Energy of propagating electromagnetic waves can be fully absorbed in a thin lossy layer, but only in a narrow frequency band, as follows from the causality principle. On the other hand, it appears that there are no fundamental limitations on broadband matching of thin resonant absorbing layers. However, known thin absorbers produce significant reflections outside of the resonant absorption band. In this paper, we explore possibilities to realize a thin absorbing layer that produces no reflected waves in a very wide frequency range, while the transmission coefficient has a narrow peak of full absorption. Here we show, both theoretically and experimentally, that a thin resonant absorber, invisible in reflection in a very wide frequency range, can be realized if one and the same resonant mode of the absorbing array unit cells is utilized to create both electric and magnetic responses. We test this concept using chiral particles in each unit cell, arranged in a periodic planar racemic array, utilizing chirality coupling in each unit cell but compensating the field coupling at the macroscopic level. We prove that the concept and the proposed realization approach also can be used to create nonreflecting layers for full control of transmitted fields. Our results can have a broad range of potential applications over the entire electromagnetic spectrum including, for example, perfect ultracompact wave filters and selective multifrequency sensors.
\end{abstract}

DOI: 10.1103/PhysRevX.5.031005

\section{INTRODUCTION}

Total absorption of electromagnetic radiation requires elimination of all wave propagation channels: reflection, transmission, and scattering. Here, we study electrically thin absorbing sheets, which are of prime importance for many applications, for example, wave filtering, radar cross-section reduction, energy harvesting, sensing, and thermal emission control. It is known that incident electromagnetic energy can be nearly fully absorbed in thin layers [1-4], but only in a narrow frequency band. The absorption bandwidth of any passive layer obeys a fundamental limitation, as follows from the causality principle [5-7]. In fact, the maximal bandwidth of absorption is proportional to the layer thickness [5]. This limitation can be overcome only by using active (pumped by some external power source) structures.

On the other hand, apparently it has not been noticed before that there is no such fundamental limitation on

\footnotetext{
* Corresponding author. viktar.asadchy@aalto.fi

Published by the American Physical Society under the terms of the Creative Commons Attribution 3.0 License. Further distribution of this work must maintain attribution to the author(s) and the published article's title, journal citation, and DOI.
}

Subject Areas: Metamaterials, Optics, Plasmonics

the frequency range in which the reflection from a thin resonant absorbing layer can be made negligible. Conceptually, we do not see any reason why it would be impossible to realize a thin layer that fully absorbs the incident power in a narrow frequency band and allows the wave to freely pass through at other frequencies, thus producing no reflections at all (within the band where the layer remains electrically thin and the inclusions forming the absorber remain electrically small). The existence of such a structure does not contradict known fundamental limitations. Indeed, the classical limitation on matching bandwidth [8] applies only to lossless matching networks. The limitation on the absorption bandwidth [5] holds only for absorbers containing an impenetrable mirror. Thus, these restrictions are not applicable for the proposed lossy structure lacking a ground plane. The limitations on periodical arrays [7] concern only the transmission properties. Obviously, exploitation of the opportunity to design a resonant absorber, which is transparent outside of the absorption band, could open up a number of novel possibilities in applications, for example, in ultrathin filters for wave trapping, selective multifrequency bolometers, and sensors. Such an all-frequencies-matched resonant absorber would be "invisible" from the illuminated sides, still acting as a band-stop filter in transmission. To the best 
of our knowledge, such wideband matched thin resonant absorbers are not known. The main goal of this paper is to explore the possibility to realize such structures.

In fact, most of the known designs of thin absorbers contain a continuous metal ground plane (a mirror) behind the absorbing layer [1-4]. The mirror obviously produces nearly full reflection outside of the absorption band. Although this feature is crucial for some applications, it forbids designing resonant absorbers which are transparent outside of the absorption band. The use of a mirror reflector can be avoided in absorbers based on arrays of subwavelength Huygens' sources (so-called Huygens' metasurfaces) that possess the appropriate level of dissipative loss. Such Huygens' sources scatter secondary waves only in the forward direction (without reflection), which destructively interfere with the incident wave, yielding zero transmission. Pioneering topologies of Huygens' inclusions were introduced in Refs. [9-12]. Subsequently, Huygens' inclusions of different topologies have been used as structural elements in sheets to control transmission wave fronts [13-15]. Recently, several topologies of absorbers have been proposed based on cut wire arrays separated by a dielectric layer [16-20]. However, in all these structures, the Huygens' balance between the electric and magnetic responses (which is necessary for cancellation of the reflected waves) holds only inside a narrow-frequency band for which the dimensions have been optimized. Outside of this band, reflections appear because of prevailing excitation of either electric or magnetic modes. The physical reason for this is that the different resonant modes exhibit different frequency dispersions. The same conclusion is valid for the idea of using a resistive sheet placed in close proximity to resonating magnetic inclusions to realize absorbing layers [21]. The analysis of these solutions reveals another feature of the known designs: The absorbers have multilayered structures. Typically, they comprise at least three layers (metal-dielectric-metal). Although manufacturing of such structures is simple and inexpensive, electric and magnetic responses in such compound architectures inevitably resonate as different electric and magnetic modes. Thus, within that scenario, it is impossible to realize a resonant absorber which is reflectionless over an ultrawide frequency range.

Furthermore, it is important to note that all the polarization-insensitive absorbers, which have been proposed so far, operate only for waves incident on one side of the absorbing layer or when the two sides are illuminated by two coherent waves. The underlying reasons for this are the presence of a ground plane (in metal-backed structures) and bianisotropic effects [22,23] (in structures without a ground plane) destroying the absorption symmetry. Moreover, this asymmetry compromises the desired invisibility of the absorbers in reflection from both sides. In our previous work [22], we demonstrated that total symmetric absorption in an electrically thin layer can be achieved only if the layer is not bianisotropic (there is no electromagnetic coupling in the layer). The only exception of achieving two-sided absorption with uncompensated bianisotropy was theoretically predicted in Ref. [22]; however, in that case, the absorbing layer is polarization sensitive. Subsequently, an example of such a symmetric and polarization-sensitive absorber has been reported in Ref. [24], providing absorption only for one circular polarization (50\% of incident power).

In this paper, the possibility to create a thin resonant polarization-insensitive absorber that produces negligible reflections in an ultrawide frequency range is explored and experimentally demonstrated. We examine the physical requirements for full and symmetric (from either of the sides) absorption of incident waves and show that the ideal performance can be accomplished in a single-layer array of specifically designed helical inclusions. We show, both theoretically and experimentally, that an array of these inclusions truly operates as a Huygens' surface in a very wide frequency range, exhibiting zero reflectivity even far from the absorption band. The governing idea behind this solution is excitation of both electric and magnetic surface currents using just one resonant mode of complex-shaped inclusions.

In our single-layer design, the magnetic response of the absorber is achieved because of current loops induced in helical elements. Although in this conceptual study we consider only metasheets based on single-turn and doubleturn helices, it is important to notice that, in principle, using the same idea, one can design metasheets comprised of helices with a large number of loops in order to decrease the structure thickness. This design would allow one to build extremely thin absorbers.

\section{SYMMETRIC TOTAL ABSORPTION IN A SINGLE-LAYER ARRAY OF RESONATORS}

Consider a uniaxial (possessing uniaxial symmetry in the plane) metasurface (or, rather, a metasheet) formed by a single two-dimensional periodic array of identical subwavelength inclusions. The inclusions are both electrically and magnetically polarizable. The subwavelength size of the inclusions and the subwavelength period allow us to describe the electromagnetic response of the metasurface in terms of electric and magnetic dipole moments induced in the inclusions. The higher-order moments do not influence the reflection and transmission coefficients for plane-wave excitation. The array is illuminated by a normally incident plane wave propagating along the $-\mathbf{z}$ direction, as shown in Fig. 1. Since the array period is smaller than the wavelength of the incident radiation, the dipole moments induced in the inclusions can be modeled as surface-averaged electric and magnetic current sheets that radiate secondary plane waves in the backward and forward directions. The backward scattered waves form reflection, whereas interference of the forward scattered and the incident waves defines transmission (see Fig. 1). As shown in Ref. [25], the electric 


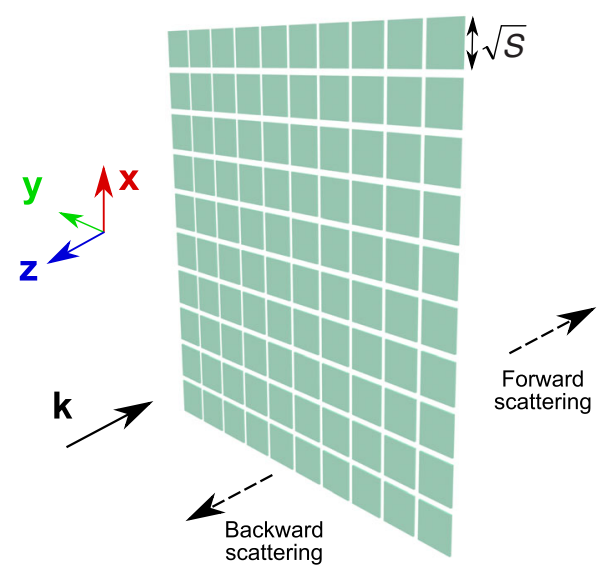

FIG. 1. A schematic illustration of a generic, electrically thin metasheet.

fields of the reflected and transmitted waves from a metasurface illuminated normally by an incident plane wave are given by

$$
\begin{aligned}
& \mathbf{E}_{\mathrm{r}}=\frac{i \omega}{2 S}\left(\eta_{0} \hat{\alpha}_{\mathrm{ee}}-\frac{1}{\eta_{0}} \hat{\alpha}_{\mathrm{mm}}\right) \mathbf{E}_{\mathrm{inc}} \\
& \mathbf{E}_{\mathrm{t}}=\left[1+\frac{i \omega}{2 S}\left(\eta_{0} \hat{\alpha}_{\mathrm{ee}}+\frac{1}{\eta_{0}} \hat{\alpha}_{\mathrm{mm}}\right)\right] \mathbf{E}_{\mathrm{inc}}
\end{aligned}
$$

where $\omega$ is the angular frequency, $S$ is the area of the array unit cell, $\eta_{0}$ is the free-space wave impedance, and $\hat{\alpha}_{\text {ee }}$ and $\hat{\alpha}_{\mathrm{mm}}$ are the effective (collective) electric and magnetic polarizabilities of the unit cells. Relations (1) hold for incident waves impinging on the metasurface from the $+\mathbf{z}$ and $-\mathbf{z}$ directions. It should be stressed that the symmetric absorption regime in a metasurface is possible only if there is no bianisotropic coupling in the structure [22]. To the best of our knowledge, all known thin single-layer absorbers demonstrate asymmetric response caused by bianisotropy of the structure. In this work, we focus on an absorber design that ideally operates for incident waves impinging on either or both of its sides and show how the bianisotropy can be suppressed.

Requiring $\mathbf{E}_{\mathrm{r}}=0$ and $\mathbf{E}_{\mathrm{t}}=0$ in Eqs. (1), one can find the conditions of symmetric total absorption in a metasurface:

$$
\eta_{0} \hat{\alpha}_{\mathrm{ee}}=\frac{1}{\eta_{0}} \hat{\alpha}_{\mathrm{mm}}=i \frac{S}{\omega} .
$$

Thus, the effective electric and magnetic polarizabilities of the unit cell normalized to the free-space impedance must be equal (corresponding to balanced electric and magnetic properties of the array) and purely imaginary (corresponding to a resonance of the inclusions in the array). This is a very important fact, meaning that in order to totally absorb incident electromagnetic radiation, the structure must possess equally significant electric and magnetic properties, and that both electric and magnetic dipolar responses of the array must resonate at the same frequency.

From Eqs. (1), the condition of perfect matching of the absorber (no reflections) reads

$$
\eta_{0} \hat{\alpha}_{\mathrm{ee}}=\frac{1}{\eta_{0}} \hat{\alpha}_{\mathrm{mm}}
$$

Here, we show that it is possible to realize a lossy and resonant array of inclusions so that the condition of full absorption (2) is satisfied at one frequency, but the condition of zero reflection (3) holds in an ultrawide frequency range, although both polarizabilities are frequency dispersive.

For the design of unit-cell topologies, it is more convenient to work with the individual polarizabilities of the inclusions in free space $\alpha_{\mathrm{ee}}$ and $\alpha_{\mathrm{mm}}$, which are related to the effective ones as [25]

$$
\frac{1}{\eta_{0} \alpha_{\mathrm{ee}}}=\frac{1}{\eta_{0} \hat{\alpha}_{\mathrm{ee}}}+\frac{\beta_{\mathrm{e}}}{\eta_{0}}, \quad \frac{1}{\alpha_{\mathrm{mm}} / \eta_{0}}=\frac{1}{\hat{\alpha}_{\mathrm{mm}} / \eta_{0}}+\frac{\beta_{\mathrm{e}}}{\eta_{0}},
$$

where $\beta_{\mathrm{e}}$ is the interaction constant of the infinite periodic array of electric dipoles. Using the known expression for the interaction constant [26], we can find the required individual polarizabilities of the inclusions of a symmetric absorber:

$$
\frac{1}{\eta_{0} \alpha_{\mathrm{ee}}}=\frac{1}{\alpha_{\mathrm{mm}} / \eta_{0}}=\operatorname{Re}\left(\frac{\beta_{\mathrm{e}}}{\eta_{0}}\right)-i \frac{\omega k^{2}}{6 \pi}-i \frac{\omega}{2 S},
$$

where $k$ is the free-space wave number. As one can see from Eq. (5), the required normalized individual electric and magnetic polarizabilities of each unit cell must be equal. Moreover, the complex quantity on the right-hand side of Eq. (5) reveals that the frequency at which the inclusions resonate in the absorbing array differs from the resonance frequency of one single inclusion in free space.

To the best of our knowledge, all known thin absorbers can be divided into two kinds, on the basis of the method of their matching. Absorbers of the first kind consist of unit cells, each of which supports electric and magnetic dipole modes resonating at different frequencies $\omega_{\mathrm{e}}$ and $\omega_{\mathrm{m}}$, respectively. The unit cell may consist of one single or two separate inclusions (for example, an electrically polarizable straight piece of metal wire and a magnetically polarizable split-ring resonator). According to Eq. (5), at the perfect-absorption frequency $\omega_{\mathrm{a}}$, the inclusions should have equal complex polarizabilities. This equality is illustrated in Fig. 2(a). Here, we model the polarizabilities using the conventional Lorentz dispersion model, which, near the resonance, adequately describes electric and magnetic dipolar response of small inclusions of arbitrary nature: 


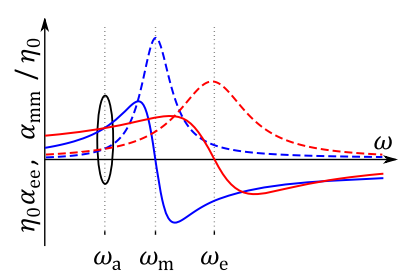

(a)

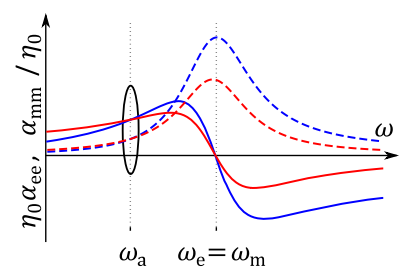

(b)
FIG. 2. Illustration of two absorption regimes in a metasurface with unit cells containing electrically and magnetically polarizable inclusions resonating (a) at different frequencies and (b) at the same frequency. Red and blue lines depict, respectively, normalized electric and magnetic polarizabilities. Solid and dashed lines show the real and imaginary parts of the polarizabilities, respectively. Calculation parameters: (a) $\omega_{\mathrm{m}}=$ $0.86 \omega_{\mathrm{e}}, A_{\mathrm{e}}=1.28 \omega_{\mathrm{e}}^{2}, A_{\mathrm{m}}=1, \gamma_{\mathrm{e}}=0.2 \omega_{\mathrm{e}}, \gamma_{\mathrm{m}}=0.1 \omega_{\mathrm{m}}, \omega_{\mathrm{a}}=$ $0.74 \omega_{\mathrm{e}} ; \quad(\mathrm{b}) \quad \omega_{\mathrm{e}}=\omega_{\mathrm{m}}, \quad A_{\mathrm{e}}=\omega_{\mathrm{e}}^{2}, \quad A_{\mathrm{m}}=1.56, \quad \gamma_{\mathrm{e}}=0.2 \omega_{\mathrm{e}}$, $\gamma_{\mathrm{m}}=0.2 \omega_{\mathrm{m}}, \omega_{\mathrm{a}}=0.8 \omega_{\mathrm{e}}$.

$$
\begin{aligned}
\alpha_{\mathrm{ee}} & =\frac{A_{\mathrm{e}}}{\omega_{\mathrm{e}}^{2}-\omega^{2}-i \omega \gamma_{\mathrm{e}}}, \\
\alpha_{\mathrm{mm}} & =\frac{A_{\mathrm{m}} \omega^{2}}{\omega_{\mathrm{m}}^{2}-\omega^{2}-i \omega \gamma_{\mathrm{m}}},
\end{aligned}
$$

where $\gamma_{\mathrm{e}}$ and $\gamma_{\mathrm{m}}$ are the loss factors of the electric and magnetic modes of the unit cell, respectively; $A_{\mathrm{e}}$ and $A_{\mathrm{m}}$ are the amplitude coefficients. Obviously, the electric and magnetic responses of the unit cell tuned for one specific frequency become unbalanced at other frequencies. This imbalance inevitably yields nonzero reflectivity of the absorber at frequencies outside of the operational band since condition (3) is satisfied only at one frequency. Typical absorbers possessing such properties have been reported in Refs. [16,17,19].

Absorbers of the second kind have a unit cell comprising one single or two separate inclusions which support electric and magnetic dipole modes resonating at the same frequency $\omega_{\mathrm{e}}=\omega_{\mathrm{m}}$. However, also in this case, in practice, it is possible to make the two polarizabilities equal only at one single frequency, while at other frequencies the different polarizabilities result in undesired reflection from the absorbing structure [see Fig. 2(b)]. Although, in this case, the two dispersion curves can be close to each other in some frequency range, in practice it is impossible to match the two polarizabilities exactly. The physical reason for this is that there is not enough design freedom in shaping the two orthogonal modes of the same unit cell so that all six main parameters (resonance frequencies $\omega_{\mathrm{e}, \mathrm{m}}$, amplitudes $A_{\mathrm{e}, \mathrm{m}}$, and loss factors $\gamma_{\mathrm{e}, \mathrm{m}}$ ) exactly match each other. Moreover, the frequency dependencies of the loss factors $\gamma_{\mathrm{e}}$ and $\gamma_{\mathrm{m}}$ are inevitably different. We can conclude that, also in thin absorbers of this kind, at least moderate reflection appears outside of the absorption band (see examples in Refs. $[20,21]$ ). Note that the problem of the unbalanced dispersion curves of the electric and magnetic modes is inherent also in all known low-loss resonators used for wave manipulations, for example, core-shell nanoparticles [27,28] and dielectric cubes [29]. Designing nonuniform arrays of such resonators reveals additional obstacles: the loss factors of the two modes inevitably have different dispersions because the radiation damping factor for a small electric dipole behaves as $\omega^{2}$, while for a magnetic dipole, the dependence is $\omega^{4}$. These obstacles result in undesired scattering from the array at all nonresonant frequencies.

To overcome the described drawbacks, we propose using inclusions designed in such a manner that both electric and magnetic responses are created by excitation of the same resonant mode. The unique feature of the proposed new metasheets is that they possess zero reflectivity over an ultrawide frequency range and are totally transparent (without polarization conversion) outside of the resonant band. The only known alternative approach to realization of all-frequency matched narrow-band absorbers is a theoretical idea of using a slab made of a lossy material with equal values of the relative permittivity and permeability in a wide frequency range [30]. Besides the fact that such media do not exist, even from the conceptual point of view, this would require a slab of infinite thickness in order to ensure zero transmission at the frequency of absorption.

\section{TOTAL ABSORPTION IN AN ARRAY OF HELICES}

Based on the above considerations, we propose using a resonant mode of a single inclusion which is coupled to both electric and magnetic fields. The induced current distribution of this resonant mode should be such that both electric and magnetic moments are excited and can be tuned to the desired balance. These properties are typical for bianisotropic elements. However, as already discussed, to behave as a symmetric single-layer perfect absorber, the structure must not be bianisotropic. In order to surmount this obstacle, we propose an unprecedented route: using bianisotropic inclusions on the level of the unit cell but arranging the inclusions in the array so that the bianisotropy is compensated on the level of the entire array. We achieve this by alternating bianisotropic inclusions of two sorts in the array. These two sorts differ only by the sign of the electromagnetic coupling parameter; therefore, their combination yields bianisotropy compensation. Thus, keeping zero electromagnetic coupling within the array, at the same time, we create coupled electric and magnetic dipole moments in each unit cell resonating exactly at the same frequency and having the same loss factors. Since the inclusions of the two sorts possess identical dipole moments, no splitting of the resonance frequency of the structure occurs.

A possibility to engineer helices so that both amplitude and loss factors in the dispersion rules (6) and (7) are equal 
can be seen from the antenna model of canonical wire helices [31]. Equal amplitudes can be ensured simply by properly choosing the helix dimensions. The loss factors $\gamma_{\mathrm{e}}$ and $\gamma_{\mathrm{m}}$ of the helix are identical because both electric and magnetic polarizabilities depend on the sum of the radiation resistances of a small electric dipole and a small magnetic dipole excited in the helix [31]. Thus, the electric and magnetic polarizabilities of the inclusions have nearly identical dispersions, and the proposed array of the inclusions acts as a Huygens' surface in a very wide frequency range. Deviations from the balance of the electric and magnetic polarizabilities caused by different frequency dependences in Eqs. (6) and (7) occur only far from the resonance, where the array is very weakly excited and reflections from it are negligible.

Although in this paper we use bianisotropic elements for wide-band reflectionless resonant absorbers, they can also be utilized for various other devices, for example, transmit arrays [13-15]. In this paper, we apply and experimentally validate our concept of all-frequency-matched Huygens' metasurfaces to absorbers in the microwave frequency range. However, the concept is generic and can be applied over the entire electromagnetic spectrum.

Here, we propose using chiral [23] bianisotropic elements as inclusions of an absorber, although one can realize similar scenarios based on other types of bianisotropic elements. There are many different topologies of chiral elements: for example, helices [11], chiral split-ring resonators [32], and cut-wire stacks [33]. In view of the simplicity of the design and realization, we utilize smooth helical inclusions [34] [see Figs. 3(a) and 3(b)]. Arrays of such inclusions operating at infrared frequencies can be

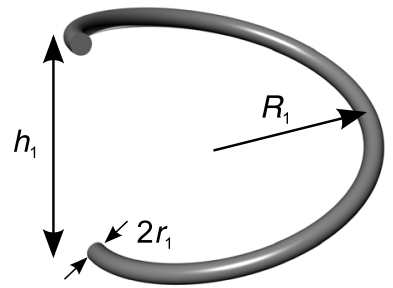

(a)

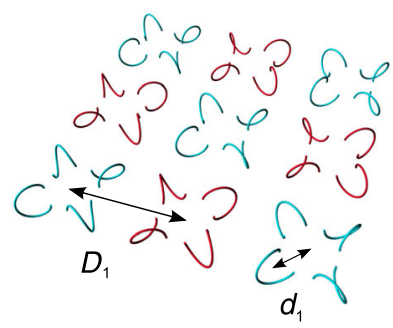

(c)

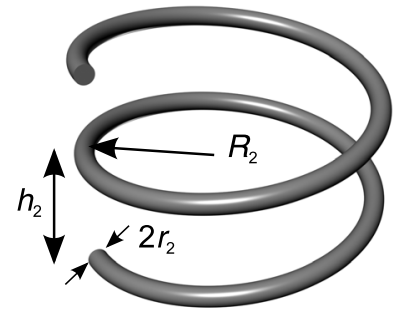

(b)

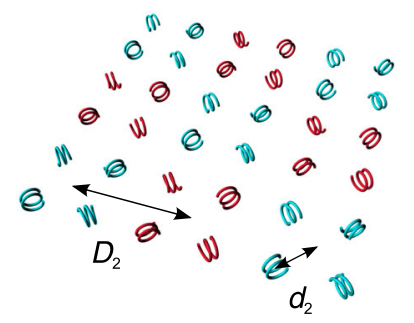

(d)
FIG. 3. (a) Single-turn and (b) double-turn helical inclusions. (c) Arrangement of the single-turn and (d) double-turn helical inclusions in the arrays. Blue and red colors denote right- and left-handed helices, respectively. manufactured based on fabrication technologies reported in Refs. [35,36]. We consider single-turn and doubleturn wire helices as two fundamental classes of helical elements. Other helices with a higher odd (even) number of loops have properties similar to those of a single-turn (double-turn) helix [37]. Because of coupled electric and magnetic moments, single-turn and double-turn helices have subwavelength dimensions $\lambda / 7$ and $\lambda / 15$ at the operating frequency, respectively. This ensures homogenization of the layer response and decreases its thickness. Compensation of chiral bianisotropy in an absorber can be achieved by alternating left- and right-handed helices.

We position the helices in the $x y$ plane in such a manner that the array has the fourfold rotational symmetry about the propagation $z$ axis and, therefore, is polarization insensitive. An optimal arrangement of helices in the array was considered in Ref. [38] and depicted in Figs. 3(c) and 3(d). The super unit cell of the array consists of four blocks of helices. The blocks comprising four helices of specific handedness are staggered in the array. The size of such a structural block in the case of single-turn helices is $D_{1}=0.6 \lambda=56 \mathrm{~mm}$, and in the case of doubleturn helices, it is $D_{2}=0.4 \lambda=43 \mathrm{~mm}$. The single- and double-turn helices are located, respectively, at distances $d_{1}=0.2 \lambda=19.8 \mathrm{~mm}$ and $d_{2}=0.2 \lambda=15.1 \mathrm{~mm}$ from the centers of the corresponding blocks. Thus, the sizes of the super unit cell in the case of single- and double-turn helical arrays are $2 D_{1}=1.2 \lambda$ and $2 D_{2}=0.8 \lambda$, respectively. Although such periodicity is not truly subwavelength, the arrays are in fact metasurfaces since they can be homogenized. Numerical simulations show that the amplitudes of the Floquet modes that become propagating for oblique illumination of the arrays are negligible compared to that of the fundamental harmonic. Therefore, these arrays can be modeled as sheets of homogeneous surface electric and magnetic currents.

Individual electric $\alpha_{\mathrm{ee}}$ and magnetic $\alpha_{\mathrm{mm}}$ polarizabilities of the helices can be made equal according to requirements (5) by varying the geometry of the helices. Using analytical expressions [12,31,37] and the method for extracting polarizabilities introduced in Ref. [39], we have optimized the dimensions of the inclusions. For single-turn helices, the loop radius is $R_{1}=7.2 \mathrm{~mm}$, the height of the loop is $h_{1}=11.3 \mathrm{~mm}$, and the wire radius $r_{1}=0.1 \mathrm{~mm}$. The corresponding parameters of the double-turn helices are as follows: $R_{2}=3.3 \mathrm{~mm}, h_{2}=2.3 \mathrm{~mm}$, and $r_{2}=0.25 \mathrm{~mm}$. The design frequency of $3 \mathrm{GHz}$ corresponds to the halfwavelength resonance frequency of the helices. The helical inclusions are embedded in a plastic foam substrate (for mechanical support) with $\epsilon=1.03$ and the thickness $14.4 \mathrm{~mm}$.

The full-absorption regime is accomplished in the array with a proper level of dissipative loss in the helical inclusions. The absorption level $A$ calculated as $1-R-T$ ( $R$ and $T$ are the power reflection and transmission 


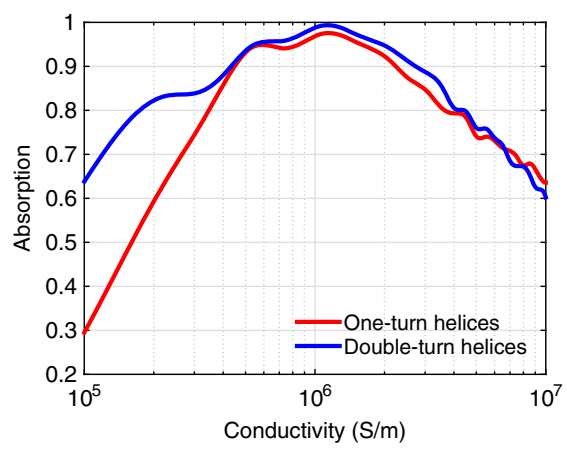

FIG. 4. Absorption coefficient versus conductivity of the inclusion material at the operating frequency of $3 \mathrm{GHz}$.

coefficients, respectively) versus conductivity of the material of the inclusions obtained with a commercial electromagnetic software [40] is plotted in Fig. 4. We see that larger or smaller than the optimum levels of dissipative loss in the inclusions degrade the performance of the absorbers. In our design, we utilize helices made of nichrome NiCr60/15 with the conductivity $10^{6} \mathrm{~S} / \mathrm{m}$, which approximately ensures the required level of dissipation loss (see Fig. 4).

The individual polarizabilities of the single-turn and double-turn helices satisfying requirements of total absorption (5) are shown in Fig. 5. The nonzero electromagnetic polarizability $\alpha_{\mathrm{em}}$ of a single inclusion is compensated in the array of the left- and right-handed helices. We see that the electric and magnetic polarizabilities have nearly identical frequency dispersions, meaning that they possess desired electromagnetic properties of wide-band Huygens' sources. This unique feature distinguishes our absorber from known designs whose structural elements possess spectrally different dispersions of the electric and magnetic modes [see Figs. 2(a) and 2(b)].

The reflection, transmission, and absorption coefficients for infinite arrays of the described single- and double-turn helices are plotted in Figs. 6(a) and 6(b). One can see that at the resonance frequency, the designed metasurfaces based on single- and double-turn helices absorb $96.5 \%$ and $99.9 \%$ of the incident power, respectively. The absorbers, because of their symmetrical

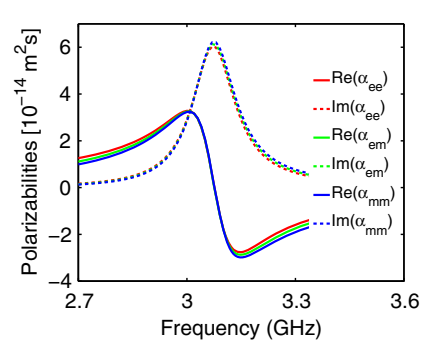

(a)

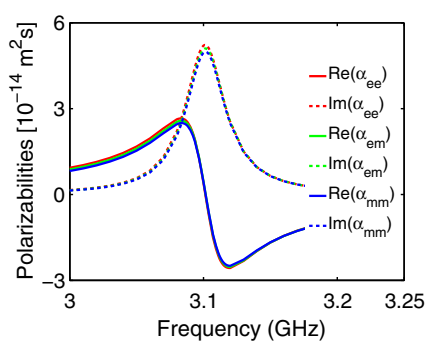

(b)
FIG. 5. Individual normalized polarizabilities of the designed (a) single- and (b) double-turn helical inclusions.

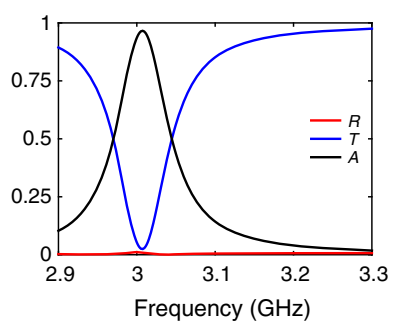

(a)

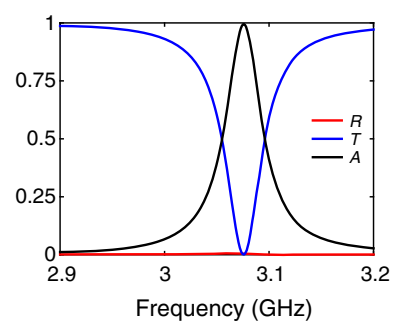

(b)

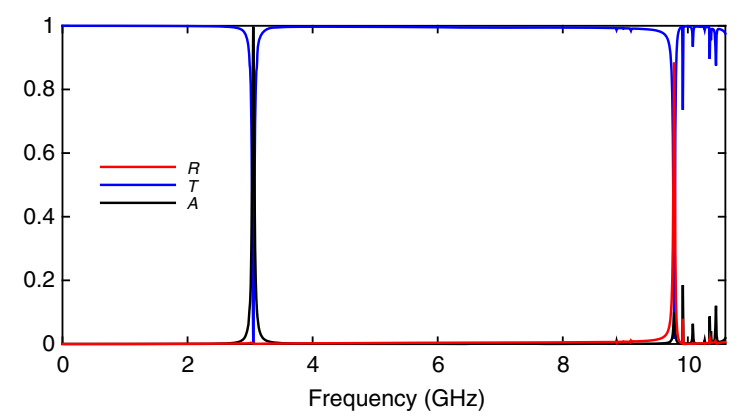

(c)

FIG. 6. Simulated power reflection $R$, transmission $T$, and absorption $A$ coefficients for infinite arrays of (a) single- and (b) double-turn helical inclusions. (c) Response of the doubleturn helix array in a wide frequency range.

geometry, identically operate with waves impinging on either of their sides. It can be seen that both inside and outside of the absorption band, the reflection coefficient is practically zero for both metasurfaces. In order to highlight the unique functionality of our absorbers, we illustrate in Fig. 6(c) the broadband response of the metasurface based on double-turn helices. While the transmission coefficient has a resonance of full absorption at about $3 \mathrm{GHz}$, the reflection coefficient is not distinguishable from zero in an ultrawide range from $\mathrm{MHz}$ frequencies to about $10 \mathrm{GHz}$, where the circumference of one turn of the helix becomes comparable to the wavelength and the first higher-order resonance appears.

The remarkable operation of the proposed Huygens' absorbers can be clearly illustrated based on a circuit analogy. To a good approximation, for frequencies not very far from the resonance, we can assume that both polarizabilities $\alpha_{\mathrm{ee}}$ and $\alpha_{\mathrm{mm}}$ of the metasheet inclusions possess the Lorentz dispersion (6). Under this assumption, the transmission coefficient (1) reads

$$
T=1+\frac{i \omega \Gamma}{\omega_{\mathrm{e}}^{2}-\omega^{2}-i \omega \Gamma},
$$

where $\Gamma=\eta_{0} A_{\mathrm{e}} / S$. Here, $A_{\mathrm{e}}$ is the coefficient from the Lorentz dispersion model (6) and $S$ is the unit-cell area of the metasheet. The reflection coefficient is identically zero at all frequencies. We can synthesize a two-port circuit with such response using the conventional circuit synthesis methods. As a result, we find that the proposed Huygens' 


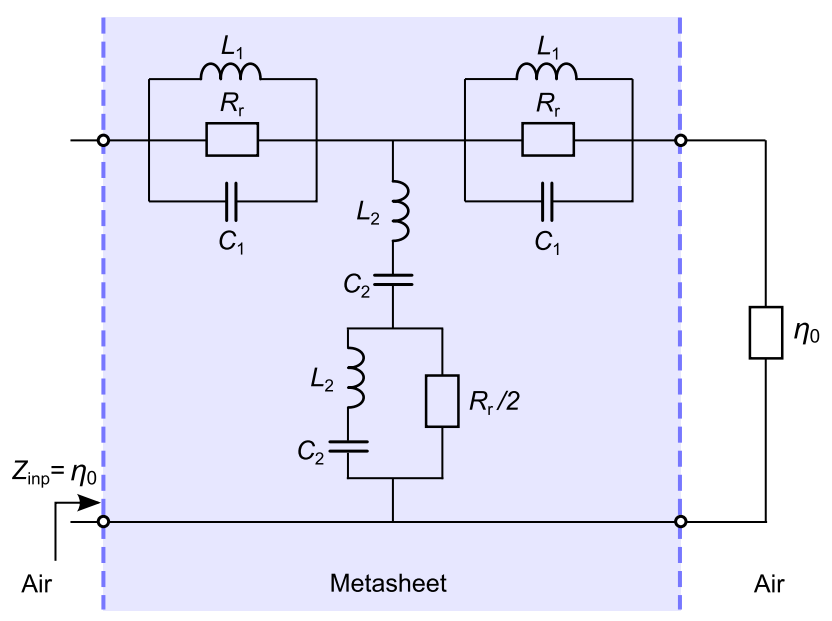

FIG. 7. An equivalent circuit of the proposed absorbers matched at all frequencies. The resistive load $\eta_{0}=377 \Omega$ denotes the impedance of free space behind the absorber.

absorbers can be modeled by the symmetric circuit shown in Fig. 7. The circuit elements in the series branches equal $1 / C_{1}=\omega_{\mathrm{e}}^{2} L_{1}=\eta_{0}^{2} A_{\mathrm{e}} / 2 S$, while those in the parallel branch are $1 / C_{2}=\omega_{\mathrm{e}}^{2} L_{2}=S \omega_{\mathrm{e}}^{2} / A_{\mathrm{e}}$. Resistance $R_{\mathrm{r}}$ is equal to $\eta_{0}$. Direct calculation of the input impedance of the absorber circuit $Z_{\text {inp }}$ reveals that it is equal to the impedance of free space $\eta_{0}$ at all frequencies (which yields zero reflection from the absorber). In the circuit theory, such circuits are known and called constant-resistance networks $[41,42]$, but it appears that mostly lossless circuits have been studied and used. Although the circuit responds as a single resistor, it is dispersive and lossy, and the transmission coefficient has a resonant behavior. At the resonance frequency $\omega_{\mathrm{e}}$, the parallel branch is short-circuited; therefore, all the incident power is dissipated in the resistor $R_{\mathrm{r}}$ in the first series branch (the metasurface totally absorbs the incident wave). At very low or very high frequencies, the series branches are short-circuited, while the parallel branch is an open circuit. Therefore, all the incident power without loss is delivered to the load resistor $\eta_{0}$, which models the free space behind the metasheet (the metasheet becomes invisible for electromagnetic radiation at these frequencies).

For many applications of absorbers, it is of particular importance to absorb normally incident radiation as well as radiation impinging on the structure at oblique angles. The angular stability of the proposed absorbers is shown in Fig. 8. It is seen that whereas the metasurface with singleturn helices absorbs nearly perfectly only at the incidence angles close to normal, the metasurface with double-turn helices exhibits impressive angular-stable absorption. This absorption can be explained by the fact that the double-turn helices have more isotropic topologies. For transverse electric (TE) polarized waves illuminating the metasurface with the double-turn helices at angles from 0 to $68^{\circ}$, the absorption level remains above $80 \%$. Furthermore, for both

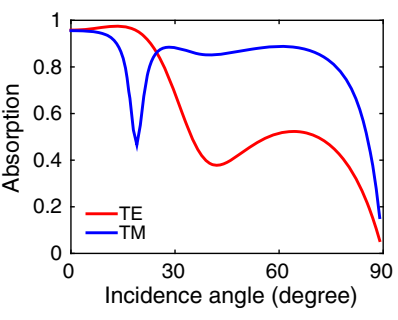

(a)

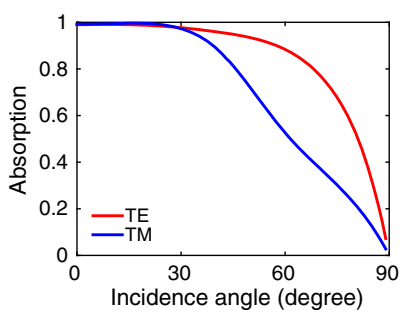

(b)
FIG. 8. The absorption level $A=1-R-T$ in a metasurface with (a) single-turn and (b) double-turn helical elements versus the incident angle.

TE and TM polarizations, the absorber operates with efficiency over $95 \%$ at angles from 0 to $35^{\circ}$. It should be stressed that this high-performance angular stability is achieved in an electrically ultrathin $(\lambda / 15)$ structure.

\section{EXPERIMENTAL REALIZATION AND CHARACTERIZATION}

The operation of the two proposed Huygens' metasheet absorbers was verified by conducting measurements in an anechoic chamber based on the free-space method [43]. A microwave signal generator was connected to a transmitting horn antenna, while the signal at the receiving horn antenna was analyzed with a microwave receiver. The horn antennas had apertures of $35 \mathrm{~cm} \times 27 \mathrm{~cm}$. The samples were located at a distance of 4 meters (about $40 \lambda$ ) from the transmitting antenna, which approximately secures plane-wave excitation of the samples. The receiving horn antenna was positioned immediately behind the samples. The fabricated samples were mounted in the hole of a wall made of microwave absorbing material. Transmission measurements were calibrated to the transmission between the horn antennas in the absence of the samples. The reflection measurements were calibrated by using a copper plate with the area equal to that of the samples. In the reflection measurements, the transmitting and receiving antennas were each inclined with an angle of about $3^{\circ}$ with respect to the normal of the sample surface.

The helical inclusions of the samples were manually manufactured and placed in supporting low-permittivity slab described in the previous section (see Fig. 9). Manufacturing single-turn helices with a small wire radius $r_{1}=0.1 \mathrm{~mm}$ optimized in simulations implies practical difficulties associated with very flexible and unstable wire forming the helix. Therefore, for our measurements, we fabricated an array of single-turn helices with the wire radius $r_{1}=0.25 \mathrm{~mm}$. The samples with single-turn and double-turn helices had dimensions of $6 \lambda \times 7.2 \lambda$ and $3.6 \lambda \times 3.6 \lambda$, respectively.

The measured power reflection $R$, transmission $T$, and absorption $A$ coefficients of the fabricated samples are depicted in Fig. 10 together with the corresponding simulated quantities (in these simulations, the single-turn 


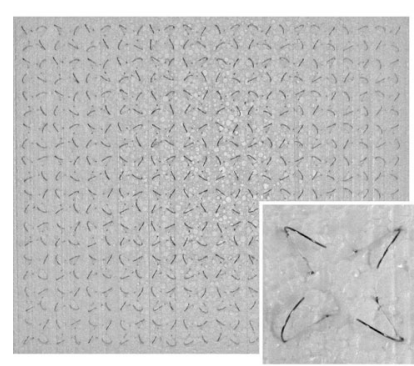

(a)

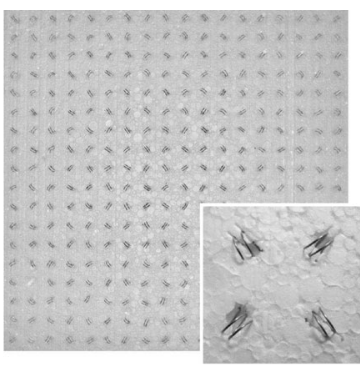

(b)
FIG. 9. Fabricated metasurfaces of (a) single-turn and (b) double-turn helices comprising, respectively, 480 and 324 elements embedded in plastic foam.

helices had the increased radius of the wire $r_{1}=0.25 \mathrm{~mm}$, the same as in the experimental sample). The absorption coefficient was found based on the reflection and transmission data using the expression $A=1-R-T$. For an array of single-turn helices, the measured peak absorption amounts to $92 \%$, while for the array of double-turn helices, the corresponding quantity achieves $81 \%$. The experimental and simulated curves plotted in Fig. 10 have similar shapes and nearly the same resonance frequencies. Because of inaccuracies in manual fabrication, the manufactured samples comprise inclusions with slightly varying geometrical dimensions such as the loop radius and the height. As a result, individual inclusions resonate at slightly different frequencies, which inevitably increases the absorption bandwidth of the structures but decreases the absorption peak values. Another reason for the resonance widening is diffuse scattering on array inhomogeneities (slightly varying period, etc.). All these imperfections can be improved with a more precise fabrication process of the helical inclusions. Both the experimental and simulated results manifestly confirm the fact that the proposed absorbers indeed operate as broadband Huygens' metasurfaces, being nearly totally reflection-free inside and outside of the absorption band.

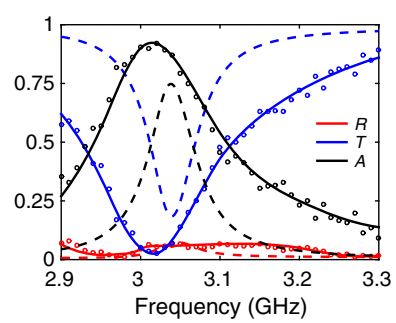

(a)

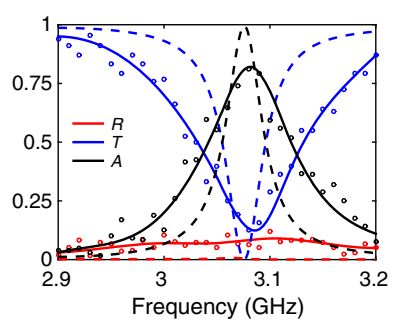

(b)
FIG. 10. Measured and the corresponding simulated reflection, transmission, and absorption coefficients for the fabricated metasurfaces with (a) single- and (b) double-turn helical inclusions. The dots denote measured values. The solid lines are the envelope curves of the measured data. The dashed lines show the corresponding simulated coefficients of the measured samples.

\section{DISCUSSION AND CONCLUSIONS}

In this paper, we have proposed and experimentally tested a novel concept of ultrathin all-frequencies-matched metasurfaces that produce no reflections in an extremely wide frequency range. Although here we utilized these metasurfaces for designing absorbers, they can be perfect candidates for creating lossless transmit arrays that are reflectionless at all frequencies and allow full control over transmission wave fronts. Indeed, let us consider a lossless wideband reflectionless metasurface that can be realized using the same helical inclusions made of a low-loss material. Assuming the Lorentz polarizability model (6) for both $\alpha_{\mathrm{ee}}$ and $\alpha_{\mathrm{mm}}$, the effective polarizabilities of lossless balanced inclusions take the form

$$
\frac{1}{\eta_{0} \hat{\alpha}_{\mathrm{ee}}}=\frac{1}{\hat{\alpha}_{\mathrm{mm}} / \eta_{0}}=\frac{\omega_{\mathrm{e}}^{2}-\omega^{2}}{A_{\mathrm{e}}}-\frac{i \omega}{2 S}
$$

as follows from (4) and (5). The amplitude of the transmission coefficient is identically equal to unity, but its phase

$$
\operatorname{phase}\left(E_{\mathrm{t}} / E_{\text {inc }}\right)=2 \arctan \left(\frac{\omega}{2 S} \frac{A_{\mathrm{e}}}{\omega_{\mathrm{e}}^{2}-\omega^{2}}\right)
$$

can be fully controlled over the $2 \pi$ range [the arctangent function in (10) varies over $\pi$ ] by choosing the unit-cell area $S$ and the inclusion parameters $A_{\mathrm{e}}$ and $\omega_{\mathrm{e}}$, still maintaining a zero reflection property at all frequencies. It should be noted that the presence of electric and magnetic responses in the structure is a necessary condition to cover the full $2 \pi$ range of the transmission phase. It is simple to prove that if only one of the electric and magnetic polarizabilities is nonzero, the accessible range of the transmission phase becomes 2 times smaller than that in Eq. (10). This result is in agreement with previous studies [44] where the phase control of transmission was limited by $\pi$ in structures possessing only an electric response.

Adjusting the level of dissipation loss in the inclusions, we can also fully control the amplitude of the transmitted wave. In contrast, known realizations of Huygens' metasurfaces for controlling transmitted waves [13-15] are perfectly matched only at one frequency.

In this paper, we have designed Huygens' metasheet absorbers, which fully absorb incident radiation of one frequency, being totally transparent for radiation of other frequencies. This regime implies that the inclusions of the absorbers are equally strongly polarized electrically and magnetically, in as wide a frequency range as possible. This polarization is achieved by using one and the same resonant mode of a chiral resonator to create both electric and magnetic responses with identical spectral dispersions. From the theoretical point of view, the proposed absorber is probably the first microwave realization of a dispersive and lossy structure which does not reveal its dispersive and 
lossy nature when observed in reflections at any frequency. Although we have designed the prototypes operating in the microwave frequency range, our generic concept of Huygens' metasurface absorbers can be applied over the entire electromagnetic spectrum.

The unique property of zero reflectivity in an ultrawide frequency range combined with frequency-selective absorption offers exciting possibilities in applications, allowing creation of perfect electrically thin band-stop filters for radiation of an arbitrary frequency. The off-band transparency of the absorbers allows one to construct various complex multifrequency filters, combining, in a parallel stack, metasurfaces resonating at different frequencies. The neighboring metasurfaces would not disturb the performance of one another, and the overall thickness of such a multilayer structure would still be of the order of one wavelength or less. Another exciting possibility of the implementation of the proposed absorbers lies in designing new types of "invisible" bolometers and sensors. Using the multilayer Huygens' metasurface absorber, it becomes possible to design a single bolometer that measures the power of incident radiation of different spectral lines at the same time. Moreover, the narrow-band response of the proposed absorbers makes them ideal candidates for implementation in bolometer arrays in astronomy at millimeter wavelengths. Because of the reflectionless operation, the proposed absorbers can be successfully used for stealth applications, especially for nonmetallic objects. In contrast to conventional metal-backed absorbers, they do not increase the radar cross section of the hidden object outside of the absorption band.

\section{ACKNOWLEDGMENTS}

This work was supported in part by the Nokia Foundation. The authors would like to thank Anu Lehtovuori and Mikko Honkala for helpful discussions of constant resistance circuits.

[1] N. I. Landy, S. Sajuyigbe, J. J. Mock, D. R. Smith, and W. J. Padilla, Perfect Metamaterial Absorber, Phys. Rev. Lett. 100, 207402 (2008).

[2] N. Liu, M. Mesch, T. Weiss, M. Hentschel, and H. Giessen, Infrared Perfect Absorber and Its Application as Plasmonic Sensor, Nano Lett. 10, 2342 (2010).

[3] M. Diem, T. Koschny, and C. M. Soukoulis, Wide-Angle Perfect Absorber/Thermal Emitter in the Terahertz Regime, Phys. Rev. B 79, 033101 (2009).

[4] B. A. Munk, Jaumann and Circuit Analog Absorbers in Frequency-Selective Surfaces: Theory and Design (Wiley, New York, 2000).

[5] K. N. Rozanov, Ultimate Thickness to Bandwidth Ratio of Radar Absorbers, IEEE Trans. Antennas Propag. 48, 1230 (2000).
[6] M. Gustafsson, C. Sohl, C. Larsson, and D. Sjöberg, Physical Bounds on the All-Spectrum Transmission through Periodic Arrays, Europhys. Lett. 87, 34002 (2009).

[7] M. Gustafsson, I. Vakili, S. Keskin, D. Sjöberg, and C. Larsson, Optical Theorem and Forward Scattering Sum Rule for Periodic Structures, IEEE Trans. Antennas Propag. 60, 3818 (2012).

[8] R. M. Fano, Theoretical Limitations on the Broadband Matching of Arbitrary Impedance, in Technical report 41 (Massachusetts Institute of Technology, Research Laboratory of Electronics, Massachusetts, 1948), p. 34, http://hdl .handle.net/1721.1/5007.

[9] M. Kerker, D.-S. Wang, and C. L. Giles, Electromagnetic Scattering by Magnetic Spheres, J. Opt. Soc. Am. 73, 765 (1983).

[10] I. V. Semchenko, S. A. Khakhomov, and A. L. Samofalov, Helices of Optimal Shape for Nonreflecting Covering, Eur. Phys. J. Appl. Phys. 49, 33002 (2010).

[11] E. Saenz, I. Semchenko, S. Khakhomov, K. Guven, R. Gonzalo, E. Ozbay, and S. Tretyakov, Modeling of Spirals with Equal Dielectric, Magnetic, and Chiral Susceptibilities, Electromagnetics 28, 476 (2008).

[12] I. V. Semchenko, S. A. Khakhomov, and A. L. Samofalov, Optimal Helix Shape: Equality of Dielectric, Magnetic, and Chiral Susceptibilities, Russ. Phys. J. 52, 472 (2009).

[13] F. Monticone, N. M. Estakhri, and A. Alù, Full Control of Nanoscale Optical Transmission with a Composite Metascreen, Phys. Rev. Lett. 110, 203903 (2013).

[14] C. Pfeiffer and A. Grbic, Metamaterial Huygens' Surfaces: Tailoring Wave Fronts with Reflectionless Sheets, Phys. Rev. Lett. 110, 197401 (2013).

[15] M. Decker, I. Staude, M. Falkner, J. Dominguez, D. N. Neshev, I. Brener, T. Pertsch, and Y.S. Kivshar, High-Efficiency Dielectric Huygens Surfaces, Adv. Opt. Mater. 3, 813 (2015).

[16] C. G. Hu, X. Li, Q. Feng, X. N. Chen, and X. G. Luo, Introducing Dipole-Like Resonance into Magnetic Resonance to Realize Simultaneous Drop in Transmission and Reflection at Terahertz Frequency, J. Appl. Phys. 108, 053103 (2010).

[17] H. Tao, N. Landy, C. Bingham, X. Zhang, R. Averitt, and W. Padilla, A Metamaterial Absorber for the Terahertz Regime: Design, Fabrication and Characterization, Opt. Express 16, 7181 (2008).

[18] G. R. Keiser, A. C. Strikwerda, K. Fan, V. Young, X. Zhang, and R. D. Averitt, Decoupling Crossover in Asymmetric Broadside Coupled Split-Ring Resonators at Terahertz Frequencies, Phys. Rev. B 88, 024101 (2013).

[19] Y. Cheng, H. Yang, Z. Cheng, and B. Xiao, A Planar Polarization-Insensitive Metamaterial Absorber, Photon. Nanostr. Fundam. Appl. 9, 8 (2011).

[20] N. I. Landy, C. M. Bingham, T. Tyler, N. Jokerst, D. R. Smith, and W. J. Padilla, Design, Theory, and Measurement of a Polarization-Insensitive Absorber for Terahertz Imaging, Phys. Rev. B 79, 125104 (2009).

[21] F. Bilotti, A. Toscano, K. B. Alici, E. Ozbay, and L. Vegni, Design of Miniaturized Narrowband Absorbers Based on Resonant-Magnetic Inclusions, IEEE Transactions on Electromagnetic Compatibility 53, 63 (2011). 
[22] Y. Ra'di, V.S. Asadchy, and S. A. Tretyakov, Total Absorption of Electromagnetic Waves in Ultimately Thin Layers, IEEE Trans. Antennas Propag. 61, 4606 (2013).

[23] A. N. Serdyukov, I. V. Semchenko, S. A. Tretyakov, and A. Sihvola, Electromagnetics of Bi-anisotropic Materials: Theory and Applications (Gordon and Breach Science, Amsterdam, 2001).

[24] M. Li, L. Guo, J. Dong, and H. Yang, An Ultra-thin Chiral Metamaterial Absorber with High Selectivity for LCP and RCP Waves, J. Phys. D 47, 185102 (2014).

[25] T. Niemi, A. O. Karilainen, and S. A. Tretyakov, Synthesis of Polarization Transformers, IEEE Trans. Antennas Propag. 61, 3102 (2013).

[26] S. A. Tretyakov, Analytical Modeling in Applied Electromagnetics (Artech House, Norwood, 2003).

[27] R. Paniagua-Dominguez, F. Lopez-Tejeira, R. Marques, and J. A. Sanchez-Gil, Metallo-dielectric Coreshell Nanospheres as Building Blocks for Optical Three-Dimensional Isotropic Negative-Index Metamaterials, New J. Phys. 13, 123017 (2011).

[28] D. Morits and C. R. Simovski, Isotropic Negative Refractive Index at Near Infrared, J. Optics 14, 125102 (2012).

[29] S. Campione, L. I. Basilio, L. K. Warne, and M. B. Sinclair, Tailoring Dielectric Resonator Geometries for Directional Scattering and Huygens Metasurfaces, Opt. Express 23, 2293 (2015).

[30] C. M. Watts, X. Liu, and W. J. Padilla, Metamaterial Electromagnetic Wave Absorbers, Adv. Mater. 24, OP98 (2012).

[31] S. A. Tretyakov, F. Mariotte, C. R. Simovski, T. G. Kharina, and J.-P. Heliot, Analytical Antenna Model for Chiral Scatterers: Comparison with Numerical and Experimental Data, IEEE Trans. Antennas Propag. 44, 1006 (1996).

[32] B. Wang, J. Zhou, T. Koschny, M. Kafesaki, and C. M. Soukoulis, Chiral Metamaterials: Simulations and Experiments, J. Opt. A: Pure Appl. Opt. 11, 114003 (2009).

[33] Y. Zhao, M. A. Belkin, and A. Alù, Twisted Optical Metamaterials for Planarized Ultrathin Broadband Circular Polarizers, Nat. Commun. 3, 870 (2012).

[34] I. A. Faniayeu, V. S. Asadchy, T. A. Dziarzhauskaya, I. V. Semchenko, and S. A. Khakhomov, A Single-Layer
Meta-Atom Absorber, in Proceedings of the 8th International Congress on Advanced Electromagnetic Materials in Microwaves and Optics, 2014 (IEEE, New York, 2014), p. 112.

[35] J. K. Gansel, M. Thiel, M. S. Rill, M. Decker, K. Bade, V. Saile, G. Freymann, S. Linden, and M. Wegener, Gold Helix Photonic Metamaterial as Broadband Circular Polarizer, Science 325, 1513 (2009).

[36] A. Radke, T. Gissibl, T. Klotzbücher, P. V. Braun, and H. Giessen, Three-Dimensional Bichiral Plasmonic Crystals Fabricated by Direct Laser Writing and Electroless Silver Plating, Adv. Mater. 23, 3018 (2011).

[37] I. V. Semchenko, S. A. Khakhomov, and A. L. Samofalov, Transformation of the Polarization of Electromagnetic Waves by Helical Radiators, J. Commun. Technol. Electron. 52, 850 (2007).

[38] V. S. Asadchy, I. A. Faniayeu, Y. Ra'di, I. V. Semchenko, and S. A. Khakhomov, Optimal Arrangement of Smooth Helices in Uniaxial 2D-Arrays, in Proceedings of the 7th International Congress on Advanced Electromagnetic Materials in Microwaves and Optics, 2013 (IEEE, New York, 2013), p. 244.

[39] V. S. Asadchy, I. A. Faniayeu, Y. Ra'di, and S. A. Tretyakov, Determining Polarizability Tensors for an Arbitrary Small Electromagnetic Scatterer, Photon. Nanostr. Fundam. Appl. 12, 298 (2014).

[40] ANSYS HFSS, 2014: www.ansoft.com.

[41] O. J. Zobel, Distortion Correction in Electrical Circuits with Constant Resistance Recurrent Networks, Bell Syst. Tech. J. 7, 438 (1928).

[42] E. L. Norton, Constant Resistance Networks with Applications to Filter Groups, Bell Syst. Tech. J. 16, 178 (1937).

[43] D. K. Ghodgaonkar, V. V. Varadan, and V. K. Varadan, A Free-Space Method for Measurement of Dielectric Constants and Loss Tangents at Microwave Frequencies, IEEE Trans. Instrum. Meas. 38, 789 (1989).

[44] L. Lin, X. M. Goh, L. P. McGuinness, and A. Roberts, Plasmonic Lenses Formed by Two-Dimensional Nanometric Cross-Shaped Aperture Arrays for Fresnel-Region Focusing, Nano Lett. 10, 1936 (2010). 